\title{
An adaptive weighting based on modified DOP for collaborative indoor positioning
}

\author{
Hao Jing1, James Pinchin2, Chris Hill1 and Terry Moore1 \\ 1(Nottingham Geospatial Institute, University of Nottingham, United Kingdom) \\ 2(Horizon Digital Economy Research, University of Nottingham, United Kingdom) (E-mail: \\ lgxhj2@nottingham.ac.uk)
}

\begin{abstract}
:
Indoor localisation has always been a challenging problem due to poor Global Navigation Satellite System (GNSS) availability in such environments. While inertial measurement sensors have become popular solutions to indoor positioning, it suffers large drifts after initialisation. Collaborative positioning enhances positioning robustness by integrating multiple localisation information, especially relative ranging measurements between local users and transmitters. However, not all ranging measurements are useful throughout the whole positioning process and integrating too much data will increase the computation cost. To enable a more reliable positioning system, an adaptive collaborative positioning algorithm is proposed which selects units for the collaborative network and integrates ranging measurement to constrain inertial measurement errors. The algorithm selects the network adaptively from three perspectives: the network geometry, the network size and the accuracy level of the ranging measurements between the units. The collaborative relative constraint is then defined according to the selected network geometry and anticipated measurement quality. In the case of trials with real data, the positioning accuracy is improved by $60 \%$ by adjusting the range constraint adaptively according to the selected network situation, while also improving the system robustness.
\end{abstract}

\section{INTRODUCTION}

Location-based Services (LBS) have gradually expanded from military and government departments into our everyday life. From emergency responders to social networks, LBS users inevitably demand for more accurate and reliable positioning information in a wider range of areas. Although Global Navigation Satellite Systems (GNSS) can provide accurate positioning outdoors, but lacks the same accuracy and robustness in more complicated environments due to signal disruption and blockage, e.g. inside buildings and urban canyons (von Watzdorf, 2010).

Inertial navigation is a common approach in GNSS-denied environments, as it does not rely on any infrastructure other than an inertial measurement unit (IMU) that works in almost any environment. However they are notorious for gyro heading drifts, which could accumulate up to tens of metres after just a few seconds. Therefore either corrections or external measurements must be applied to inertial measurements to provide more reliable results (Abdulrahim et al., 2011). Various inertial navigation system (INS) integration methods, such as INS/GPS and INS/Wi-Fi integration, have been proposed where each sensor complement the other if one fails during a short period (Evennou and Marx, 2006; Weyn and Schrooyen, 2008). Wireless signals are available indoors and naturally become a good alternative indoors, even though it suffers signal instability (Narzullaev et al., 2008; Kaemarungsi and Krishnamurthy, 2012).

3 Collaborative positioning integrates multiple systems into a single network, which was first 
introduced in intelligent transport systems. Roadside beacons and vehicle clusters helped to maintain reliable positioning by correcting GNSS observations and reduce errors through vehicle-to-vehicle ranging when the vehicle could not receive sufficient satellite signals (Yao et al., 2011; Tang et al., 2012). For a more general idea of collaborative positioning, signal of opportunities was introduced in (Yang et al., 2009) where positioning is achieved from integrating a number of different types of signals in the surrounding environment. However, the overall performance can be affected by the reliability of each particular signal, the amount of data and the relative position.

This paper proposes a collaborative positioning solution for an indoor pedestrian navigation scenario, which integrates measurements from multi-users through peer-to-peer (P2P) ranging. Based on the signal properties in the indoor environment, this paper provides a detailed analysis on the collaborative network structure and its effects on the positioning result. A particle filter based adaptive ranging constraint collaborative positioning (ARCP) algorithm is proposed which integrates inertial measurements, map information and relative ranging. It improves the positioning accuracy and robustness in complicated indoor environments by applying a selecting and weighting scheme to the ranging constraint on each user based on the obtained ranging measurements and network geometry. Simulations are carried out to analyse the network characteristics and the anticipated positioning outcome. Finally, trials are carried out to validate the positioning algorithm performance.

\section{SELECTING THE NETWORK}

Multi-users can share local environment information and constrain errors directly through P2P ranging between users (Jing et al., 2013). P2P ranging are relative ranging measurements between nearby units, which can update and correct the user state model by restricting valid measurements to the measured distance and pushing the final solution towards the true position. Therefore, the ranging constraint plays a crucial role on the positioning performance. To integrate only the most effective ranges, a decision-making scheme is introduced here to enhance positioning accuracy and system efficiency.

The decision is made each epoch based on the current situation, hence is adaptable to different measurement error models and network geometry. Three different aspects are considered. First of all, the ranging measurement accuracy level estimated from signal characteristics. Secondly, the network geometry of the collaborative network formed by selected nodes. Finally, the network size should also be considered for efficiency.

The collaborative network discussed here consists of two types of units, fixed transmitters with known positions, known as anchors (denoted as Tx), and mobile users whose positions need to be determined, known as rovers (denoted as Rx). The optimal network should consist the minimum number of units that produces the required positioning accuracy. The CramerRao Lower Bound (CRLB) of different networks is presented below to examine the relationship between network size, geometry and positioning accuracy.

\section{2 \\ 2.1 Network Cramer-Rao Lower Bound}

CRLB provides a lower boundary on the achievable variance of any unbiased location estimator for unknown parameters, which is useful for justifying how well an estimator can perform (Patwari et al., 2005; Wymeersch et al., 2009; Penna et al., 2010). CRLB states that

86 the variance of an unbiased estimator $\hat{\theta}$ should at least be as high as the inverse of a function of the expectation taken with respect to the probability density function (pdf) $p(x ; \theta)$, 


$$
C R L B=\frac{1}{-E\left[\frac{\partial^{2} \ln p(x ; \theta)}{\partial \theta^{2}}\right]} \leq \operatorname{var}(\hat{\theta})
$$

106

where the derivate is evaluated at the true value of $\theta$. Assume that we are interested in ranging measurement stated as,

$$
r_{i}=\sqrt{\left(\hat{x}_{u}-x_{i}\right)^{2}+\left(\hat{y}_{u}-y_{i}\right)^{2}}+\varepsilon
$$

where $\left(\hat{x}_{u}, \hat{y}_{u}\right)$ is the user location, $\left(x_{i}, y_{i}\right)$ is the $i$ th reference node, $r_{i}$ is the ranging measurement centred at $h(\hat{\theta})$ with a noise $\varepsilon$ of Gaussian zero mean with covariance $R$. If there were $m$ nodes in the network and $H=\frac{\partial}{\partial \theta} h(\theta)$. CRLB at location $(x, y)$ is given by

$$
C R L B(x, y)=\sqrt{\operatorname{tr}\left(\left(H^{T} R^{-1} H\right)^{-1}\right)}
$$

where $R=\operatorname{diag}\left(\sigma_{1}^{2}, \sigma_{2}^{2}, \ldots, \sigma_{m}^{2}\right), \sigma_{i}^{2}$ is the variance of $i$ th measurement. The resulting CRLB indicates the positioning accuracy level at each location. The performance of different networks is discussed below in detail.

\subsection{Ranging accuracy}

Generally, ranging measurement noise $\varepsilon$ consists of white noise $w$ and bias $b$. Thus Eq.(2) can be rewritten as,

$$
r_{i}=\sqrt{\left(\hat{x}_{u}-x_{i}\right)^{2}+\left(\hat{y}_{u}-y_{i}\right)^{2}}+w_{i}+b_{i}
$$

The white noise is a zero mean variable with a variance of $\sigma^{2}$, which can be modelled based on prior observation data. To testify the error bound for range-based positioning of different measurement levels, four different anchor locations are set on each corner of a $100 \mathrm{~m} \times 100 \mathrm{~m}$ square area. The CRLB of the entire simulated area is calculated for different noise levels with variances of $\sigma^{2}=1, \sigma^{2}=3$ and $\sigma^{2}=5$ while bias $b=1 \mathrm{~m}$ and $b=5 \mathrm{~m}$ respectively. Blue indicates low CRLB and red indicates high values.

$$
\begin{array}{ll}
\text { (a) } \sigma^{2}=1, b=1 & \text { (b) } \sigma^{2}=1, b=5
\end{array}
$$

$$
\begin{array}{ll}
\text { (c) } \sigma^{2}=3, b=1 & \text { (d) } \sigma^{2}=5, b=1
\end{array}
$$

\section{Figure 1 CRLB with different noise variance and bias}

107

While CRLB increases when the variance or bias increases, meaning a lower positioning accuracy, the effect of the variance is larger than that of the bias.

\subsection{Network dilution of precision}

The accuracy of GNSS positioning at a point on Earth is related to the geometry of observable satellite constellation, which is reflected by the dilution of precision (DOP) (Langley, 1999). Thus good signal geometry plays a significant role in positioning which restricts the measurement uncertainty into a smaller boundary.

The geometry of the collaborative network can be assumed as the satellite constellation projected onto a 2-D scenario. If ranging measurements between a user located at $\left(\hat{x}_{u}, \hat{y}_{u}\right)$ and surrounding units located at $\left(x_{i}, y_{i}\right)$ is expressed as Eq.(4), the coordinate differences 
117 form the geometry matrix, $A=\left(\begin{array}{ccc}\frac{\hat{x}_{u}-x_{1}}{r_{1}} & \frac{\hat{y}_{u}-y_{1}}{r_{1}} & 1 \\ \frac{\hat{x}_{u}-x_{2}}{r_{2}} & \frac{\hat{y}_{u}-y_{2}}{r_{2}} & 1 \\ \vdots & \vdots & \vdots \\ \frac{\hat{x}_{u}-x_{m}}{r_{m}} & \frac{\hat{y}_{u}-y_{m}}{r_{m}} & 1\end{array}\right)$, where $\wedge$ denotes estimated results. In

118 2-D positioning, the horizontal DOP (HDOP) is defined as

$$
H D O P=\sqrt{\operatorname{trace}\left(\left(A^{T} A\right)^{-1}\right)}
$$

119 DOP can be applied to analyse the collaborative network from two aspects. First of all, lower 120 DOP indicates better positioning geometry. Secondly, increasing units could also potentially reduce the DOP. Figure 2 reflects that the positioning uncertainty boundary is closely affected by the relative position of the units. When the two anchors are close together, DOP increases as well as the positioning uncertainty.

\section{(a) Good geometry}

(b) Bad geometry

Figure 2 Network geometry and error boundary

\subsubsection{Network Geometry Quality}

126 In recent works, DOP has been applied to the analysis of geometric and signal strength for GPS/Wi-Fi and cellular communications positioning system (Zirari et al., 2009; Chen et al., 2013). In this paper, DOP is used as an indicator of the anticipated network performance. The corresponding CRLB and DOP is compared for the designated area described in Section 2.2 where two anchors placed at different locations along the side of the area, marked as red diamonds in Figure 3 and Figure 4. Dark blue indicates low values and red indicate high values. Results indicate that low DOP areas correspond to the low CRLB areas.
(a) $\mathbf{T x 1 , 2}$
(b) $\mathrm{Tx1} 1,4$
(c) $\mathbf{T x 1 , 5}$
(d) $\mathbf{T x 5 , 6}$

Figure 3 CRLB for different geometry settings
(a) $\mathrm{Tx1}, 2$
(b) $\mathrm{T} \times 1,4$
(c) $\mathrm{Tx1}, 5$
(d) $\mathbf{T x 5 , 6}$

\section{Figure 4 DOP for different geometry settings}

\subsubsection{Network Capacity}

134 Increasing the network size is also a solution to improving accuracy. As Yang (2014) suggests, increasing the number of units will give better positioning performance in collaborative positioning scenarios. As the network size increases, the relative location of the anchors becomes a less dominating factor. However, the number of anchors should be controlled so that computation cost is kept as low as possible without affecting the positioning performance. Therefore the number of anchors should be carefully selected to maintain the balance. In this case, a threshold should be identified where increasing the unit number begins to have less obvious impact on improving positioning performance. 
The CRLB of the designated area corresponding to network sizes increasing from two to eight are computed, of which four scenarios are shown below. The anchors are located along the side of the designated area marked as red diamonds in Figure 5, while noise level remains $\sigma^{2}=1$. Results indicate an obvious decrease in CRLB when the network size increases, however the deduction rate is reduced when the number of anchors reaches four.
(a) Tx1-3
(b) Tx1-4
(c) Tx1-5
(d) Tx1-8

Figure 5 CRLB for different network sizes

\section{3. ADAPTIVE COLLABORATIVE POSITIONING}

\section{$148 \quad 3.1$ Gathering measurements}

149 The proposed collaborative positioning aims to constrain inertial measurement errors by 150 integrating external information to a pedestrian dead reckoning (PDR) model. A popular method of constraining heading bias in indoor positioning is through map matching. P2P ranging is also integrated to provide further constraint. The principles of PDR are given below, as well as the characteristics of the other measurements.

\subsubsection{Pedestrian Dead Reckoning}

PDR obtains the current position from a relative measurement between the current state and the previous state, e.g. the distance and direction travelled. These measurements may be obtained from any inertial device that provides a step count and heading, e.g. low-cost IMUs or smartphone. The step length is usually estimated by a step recognition model or set to a constant value, e.g. $0.7 \mathrm{~m}$, and later corrected through filtering. The basic PDR model is as below,

$$
\left[\begin{array}{l}
\hat{x}_{k} \\
\hat{y}_{k}
\end{array}\right]=\left[\begin{array}{l}
\hat{x}_{k-1}+\hat{s}_{(k \mid k-1)} \cos \hat{\theta}_{(k \mid k-1)} \\
\hat{y}_{k-1}+\hat{s}_{(k \mid k-1)} \sin \hat{\theta}_{(k \mid k-1)}
\end{array}\right]
$$

161 whereas $\left[\hat{x}_{k}, \hat{y}_{k}\right]$ is the estimated position at time $k, \hat{s}_{(k \mid k-1)}$ is the estimated step length

162 from time $k-1$ to time $k, \hat{\theta}_{(k \mid k-1)}$ is the measured heading. Due to gyro drifts, the heading 163 measurement $\hat{\theta}$ tends to be biased which increases continuously if no correction is 164 implemented.

\subsubsection{Peer-to-peer Ranging}

166 Recent studies have shown that Ultra-Wideband (UWB) systems can achieve decimetre, or centimetre level ranging and positioning accuracy in an open environment (Gentile and Kik, 2007; $\mathrm{Xu}$ and Law, 2009). Hence multi-user collaborative networks can be established through P2P measurements from UWB units. Although not currently widely applied due to cost and many other reasons, the implementations of UWB in mobile devices can potentially boost the its application popularity (Seo and Lee, 2010). Furthermore, the recent advances in wireless technology brings forth Bluetooth 4.0 and $5 \mathrm{G} \mathrm{Wi-Fi,} \mathrm{both} \mathrm{of} \mathrm{which} \mathrm{has} \mathrm{greater}$ potential in providing much higher accuracy ranging estimations than current wireless signals (Cinefra, 2012).

175 UWB systems work on a bandwidth of more than $1 \mathrm{GHz}$ and spread the signal pulses along 
the whole bandwidth so that they are able to transmit signals at a very high time resolution, which enables high accuracy ranging (Lee and Scholtz, 2002; Koppanyi et al., 2014). Yet UWB measurements are also influenced by obstructions that cause non-line-of-sight (NLOS) signals, which disturb signal properties and reduce accuracy.

Many methods have been proposed to identify UWB NLOS signals and characterise its accuracy level based on signal characteristics (Ismail et al., 2008; Marano et al., 2010; Wymeersch et al., 2012). The collaborative constraint is set adaptively according to the ranging measurement and the detected accuracy level, which is converted to the anticipated standard deviation of the ranging measurement.

\subsubsection{Interior Map Information}

Map matching is commonly applied to constrain measurement errors in indoor navigation by forcing the user to stay within the reasonable path, i.e. pedestrians can only walk along corridors and travel through doors (Pinchin et al., 2012). As shown in Figure 6, the user could only enter Room 2 by going out of the door into the corridor (c1) and then go through the door linking $\mathrm{c} 1$ and Room 2. The trouble with interior maps is that they must be available prior to use.

\section{Figure 6 Implementation of room polygons}

\subsection{Particle filtering based collaborative positioning}

Particle filtering $(\mathrm{PF})$ is a recursive Bayesian filtering method that handles non-linear and non-Gaussian systems. It has been widely applied to positioning and navigation problems due to its ability to integrate different measurements (Gustafsson et al., 2002). PF predicts the system states through sequential Monte Carlo estimation from a large set of particles with associated weights that represent the state probability density function (pdf). The system state vector $x_{k}$ is a discrete time stochastic model:

$$
x_{k}=f_{k}\left(x_{k-1}, v_{k-1}\right)
$$

where $k$ is the time index, $f_{k}$ is the non-linear function of the state $x_{k-1}$ and process noise $v_{k-1}$. The state vector $x_{k}$ is recursively updated from observation $z_{k}$ :

$$
z_{k}=h_{k}\left(x_{k}, w_{k}\right)
$$

where $h_{k}$ is usually a non-linear function with measurement noise $w_{k}$. PF looks into estimating the state $x_{k}$ at time $k$, given observations $z_{1: k}$ up to time $k$. At each epoch, the predicted pdf is updated through measurements to represent the posterior pdf of the current state. However it is usually impossible to obtain the true posterior pdf. Therefore $N$ particles are generated to represent a discrete approximation $p(x)$,

$$
p(x) \approx \sum_{i=1}^{N} w^{i} \delta\left(x-x^{i}\right)
$$

207 where $w^{i}$ is the weight of the $i$ th particle. As $N \rightarrow \infty$, the approximation should approach the true posterior pdf (Arulampalam et al., 2002).

209 A basic PF based collaborative positioning (CP) is outlined below: 
i. Initialisation: generate $N_{p}$ particles around the initial position of each $\operatorname{Rx}\left[x_{0}, y_{0}\right]$, all particles are assigned an equal weight $w_{k}^{i}=1 / N_{p}$.

212 ii. Prediction: particles propagate forward based on the PDR prediction model Eq.(6). The step length is a constant value $s l$ with a uniformly distributed random noise $\mathcal{U} \sim\left(-n_{s}, n_{s}\right)$, the heading $\hat{\theta}_{(t \mid t-1)}$ consists of a constant heading bias $b_{h}$ and a uniformly distributed random noise $\mathcal{U} \sim\left(-n_{h}, n_{h}\right)$.

iii. Update and weighting: particles that cross walls are "killed", i.e. $w_{k}^{i}=0$. Collaborative constraint is then implemented by obtaining the ranging measurements $\hat{r}_{m}$ between the current rover to each other unit, as well as the distance $\hat{d}_{m}^{i}$ calculated from each particle of the rover to the other $N_{\mathrm{m}}$ units. For a particular particle $i$, if the difference between the two is over a collaborative constraint threshold thres $r$

$$
\operatorname{diff} f_{i}=\left|\hat{d}_{m}^{i}-\hat{r}_{m}\right|_{m=1,2, \ldots, N_{m}} \geq \text { thres }_{r}
$$

221

222

223

224

225

226

227

228

229

230

231

232

233

234

235

236

\section{Figure 7 Simulated positioning network}

the particle is "killed". thres $s_{r}$ is given based on the anticipated accuracy level of ranging measurements.

iv. Resampling: if the number of "live" particles falls below a threshold, i.e. $N_{p} / 2$, new particles are generated to maintain a total number of $N$ particles.

v. Return to ii or end iteration.

This algorithm is applied to the simulated network as shown in Figure 7. Eight potential locations are indicated along the sides of a square area of $100 \mathrm{~m} \times 100 \mathrm{~m}$ where anchors could be placed. North direction points upwards along the $y$-axis, East points rightwards along the $x$-axis. A single trajectory is defined and plotted in green line while the magenta line indicates the PDR result. Five different location pairs for two anchors are simulated at five different locations hence producing different geometries. Figure 8(a) indicates the positioning error of the networks with different DOPs, Figure 8(b) shows the DOP for each network while the rover is moving.

(b)

Figure 8 (a) Positioning errors for different networks (b) DOP of each network Figure 9 Positioning errors for different network sizes

To examine the positioning accuracy of different network size, the performance of networks consisting from 2 up to 10 anchors (shown in Figure 7) is examined. The mean positioning errors for different ranging accuracy levels, i.e. ranging error standard deviation $\sigma_{\text {err }}$ of 3,5 and 15 respectively, are plotted in Figure 9. We could see a distinct improvement in positioning when the number of anchors increases from three to four for networks when $\sigma_{e r r}=3$ and $\sigma_{e r r}=5 \mathrm{~m}$, and four to five when $\sigma_{\text {err }}=15$. The improvement in positioning becomes less evident after this size is reached. An increase in positioning error can actually be spotted when the number of anchors increases from 7 to 8 when $\sigma_{\text {err }}=3 \mathrm{~m}$ and $\sigma_{\text {err }}=$ $15 \mathrm{~m}$, and from 8 to 9 when $\sigma_{e r r}=5 \mathrm{~m}$. This is due to the inaccuracy in the ranging 
measurement, when the number of ranging constraint increases, so does the inaccuracy in the constraint. Hence such increases is more likely when the ranging measurement itself is uncertain, e.g. the increase happens twice when $\sigma_{\text {err }}=15 \mathrm{~m}$. Therefore, it is not a good idea to use more than the necessary number of units in a collaborative network, especially when the measurement themselves contain error or bias. Yet the optimal accuracy cannot be achieved if not enough units are used. Thus keeping a balance of the network size is important.

\subsection{Modified DOP}

Although DOP demonstrates the relationship between the geometry and positioning performance, it cannot reflect all details inside a collaborative network. The first factor that is not reflected in DOP is the ranging accuracy, which directly influences the effectiveness of the constraint in collaborative positioning. The constraint threshold thres $s_{r}$ is defined as the anticipated accuracy level of the ranging measurements plus an "error boundary". However, if this threshold is smaller than the measurement error itself, i.e. the constraint is too "tight", the positioning estimation would be pushed towards a wrong location. On the other hand, if the bound is much larger than the error, i.e. constraint too "weak", then the observation noise and error may not be sufficiently eliminated.

Therefore, a modified DOP (MDOP) that integrates the ranging quality is proposed here and the geometry matrix $A_{\text {mod }}$ is computed as below,

$$
A_{\text {mod }}=\left(\begin{array}{ccc}
\frac{\hat{x}_{u}-X_{1}}{a \cdot r_{1}} & \frac{\hat{y}_{u}-Y_{1}}{a \cdot r_{1}} & 1 \\
\frac{\hat{x}_{u}-X_{2}}{a \cdot r_{2}} & \frac{\hat{y}_{u}-Y_{2}}{a \cdot r_{2}} & 1 \\
\vdots & \vdots & \vdots \\
\frac{\hat{x}_{u}-X_{n}}{a \cdot r_{n}} & \frac{\hat{y}_{u}-Y_{n}}{a \cdot r_{n}} & 1
\end{array}\right)
$$

265

266

267

268

269

where $a$ is a measurement accuracy coefficient derived from accuracy detection. The detection method provides the user with how likely the measurement is reflecting the true distance, which is given by $a$, a value between 0 and 1 . Hence reliable measurements produce $a$ closer to 1 and $A_{\text {mod }}$ would be close to $A$. MDOP is computed from $A_{\bmod }$ as in Eq. (12), thus the produced MDOP is usually larger than the original DOP.

$$
M D O P=\sqrt{\operatorname{trace}\left(\left(A_{\text {mod }}^{T} A_{\text {mod }}\right)^{-1}\right)}
$$

While the rover is always moving during navigation, it is hard for the DOP to reflect the dynamic directional information, e.g. the direction of the bias of the current rover relative to the anchors. Figure 10 shows the error in both the East and North directions when CP is applied to the rover simulated in Figure 7. As Tx5 and Tx6 are located on either side of the rover, the network constrains the error in the North direction better than the East direction. Tx7 and Tx 8 are both located to the north of the rover, thus constrains the error in the East direction better than the North direction. Measurements coming from different directions will constrain error along different directions. The selected units should consider the dynamic situation of the rover as directions change.

\section{(a) Tx5-6 network}

\section{(b) Tx7-8 network}




\section{Figure 10 CP Positioning error of different networks}

280 MDOP is not just a value that reflects the geometry with the ranging accuracy, but rather a concept of considering all the relevant information of a dynamic collaborative network, i.e. the ranging accuracy, the network geometry, the relative positions of the units.

\section{$3.4 A R C P$}

When collaborative units are available, the appropriate units should be selected to form a network with the optimal MDOP to produce the best positioning results. The adaptive ranging constraint collaborative positioning (ARCP) method is developed here and its procedure outlined in Figure 11. Compared to CP, the adaptivity of the ARCP is defined from three aspects: adaptability to varying measurement accuracies, the flexibility to select different network size and unit locations. More specifically, the adaptivity is reflected in the selection of the appropriate units.

290

Once the rover takes a step based on PDR, it will look for local units to form the collaborative network. The optimal size of the network is considered to be four according to the simulation results presented in Section 3.2. Integrating too many or not enough units will both results in reduced positioning performance. If more than four units (including rovers and anchors) are available, the estimated accuracy level of the ranging measurement from each unit is obtained. Those units with an associated $a$ larger than 0.5 are considered as potential units. They are then combined with the current rover to form a network of four units and the MDOP of each possible network is computed. The relative positions of the units are also considered by sharing the position of the anchors and the estimated position of the other rovers. The network with the smallest MDOP value is selected as the optimal network. The constraint thres $_{r}$ for each ranging measurement is set according to MDOP, which reflects both $a$ and DOP. If less than four units are available, the units would simply be included in the collaborative network and thres ${ }_{r}$ set according to MDOP.

\section{Figure 11 Flowchart of ARCP}

$a$ can be converted to the estimated standard deviation of the measurement $\sigma_{r}$, which is then applied as the constraint threshold. $a$ is mapped onto three categories of thres,

$$
\operatorname{thres}_{r}(a)=\left\{\begin{array}{lc}
1, & a \geq 0.8 \\
2, & 0.5 \leq a<0.8 \\
3, & a<0.5
\end{array}\right.
$$

307 The values are selected based on real indoor measurement error levels and indicate the 308 expected error in metres. Most measurements should fall within category 1 or 2 . A threshold 309 of 3 indicates a very loose constraint, where the rover mostly depends on PDR propagation. 310 The thres $s_{r}$ is further derived from DOP based on Eq.(14) which multiplies a coefficient on 311 to $\operatorname{thres}_{r}(a)$. Simulations have shown that if the threshold were set to the same value as the 312 real measurement standard deviation, the constraint would be too tight. Hence the final threshold is always larger than the expected error standard deviation. The threshold categories can be adjusted but the values applied here are selected from the combination that gives the best constraint performance for the simulations in this paper.

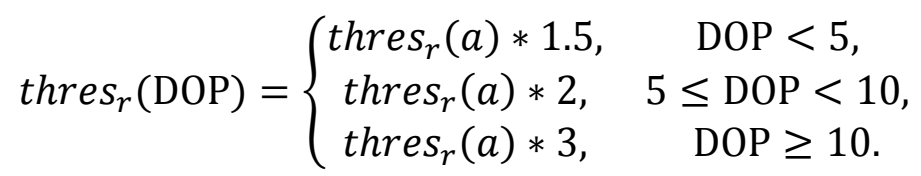


316 By applying the ARCP, the possibility of selecting units with a low ranging accuracy is

317 reduced. The constraint threshold is then also set according to the estimated measurement

318 accuracy. Hence a collaborative network consisting the optimal units will more likely output

319 positions with higher accuracy and reliability. While less optimal network positioning mostly

320 depend on inertial measurements.

321 ARCP is applied to the same networks as those in Figure 8 and the positioning error of ARCP

322 and CP is compared in Figure 12. An obvious improvement could be seen when the adaptive

323 selection is applied.

\section{Figure 12 Positioning error comparisons for $\mathrm{ARCP}$ and non-adaptive $\mathrm{CP}$}

\section{ALGORITHM EVALUATION}

\subsection{Simulations}

The proposed ARCP algorithm is applied to two sets of trials, denoted as Trial A and Trial B, to validate its application with in real environments. Data are collected and post-processed in real-time mode on Matlab 2013 and different algorithms are implemented for comparison. For both trials, the inertial data are collected using MicroStrain 3DM-GX3®-25 IMU, which is connected to a Raspberry Pi for data logging. The step and heading information are then extracted and applied to the PDR model. The interior building map was surveyed by Leica TS30 total station and loaded into Matlab as polygons (rooms and corridors) and points (doors).

In Trial A1, all real inertial data is collected in the Nottingham Geospatial Building (NGB), University of Nottingham. Three anchors ( $\mathrm{T} x 1, \mathrm{~T} \times 2$ and $\mathrm{Tx} 3$ ), are simulated at different locations inside the building to provide extra ranging constraint. The ranging data between the rovers and anchors are simulated based on indoor ranging performance of wireless signals, where the error variance is larger when the two rovers are in NLOS and smaller when there is no obstruction. The basic CP algorithm is applied in Trial A1 by integrating one of the anchors into the network. The measurement error of the rover is constrained by integrating the ranging measurement from the other rover and one anchor at every epoch by applying a constant threshold. The non-adaptive result of the network consisting Rx1, Rx2 and Tx1 is shown in Figure 13. The green line indicates the ground truth for both rovers, the cross dot line indicates the position estimation of Rover 1 and the circle dash line indicates the position estimation of Rover 2.

Figure 13 CP Positioning result with wall constraint (Trial A1-Tx1)

The ARCP algorithm is applied in Trial A2 where each rover selects one anchor to form a collaborative network with the other rover that produces the optimal MDOP at every epoch. thres $_{r}$ is adjusted according to MDOP. Results are shown in Figure 14. The plot indications are the same as Figure 13.

Figure 14 ARCP Positioning result (Trial A2)

\section{Figure 15 ARCP Positioning result without map matching (Trial A3)}


shown in Figure 15. Therefore, the particles are no longer restricted from crossing walls and the measurement error is bounded only by the ranging constraint.

\subsection{Real data}

Trial B was carried out in the Business School Building, University of Nottingham. PDR data is collected using the same equipment worn on two pedestrians, Rover 1 and Rover 2. A UWB network was setup in the building as indicated with a red star in Figure 16 to act as anchors and provide ranging measurement. Each rover also carries a mobile UWB unit to receive ranging measurements from other units.

\section{(a) Rover 1}

\section{(b) Rover 2}

\section{Figure 16 Trial B Ground truth for Rover 1 and Rover 2}

363

364

365

366

367

368

369

370

371

372

373

Data was collected for ten minutes. In every epoch, each rover selects a number of the anchors to form a collaborative network with the other rover. The network size and the ranging constraint threshold are adjusted according to the actual network quality.

The ground truth is plotted in Figure 16. Due to lack of equipment, the ground truth of Rover 2 was provided by the UWB system, whose outdoor performance is disrupted (light blue part of the trajectory in Figure 16(b)), as all units are setup indoors. The positioning result for Rover 1 and Rover 2 is shown in Figure 17 (a) and (b) respectively. The green solid line indicates the ground truth, the cyan dashed line shows the PDR output from raw inertial data. The blue line represents the ARCP output with wall constraint, and magenta line represents the ARCP result without wall constraint.

(a) Rover 1

(b) Rover 2

\section{Figure 17 ARCP Positioning result for Rover 1 and Rover 2 (Trial B)}

374

375

376

377

378

379

380

381

382

383

384

385

386

387

388

389

390

391

392

\subsection{Results}

Collaborative positioning is able to constrain measurement errors by integrating relative ranging constraint into the system. However in reality, this does not always give the best performance due to the complexity of real data, which could be caused by environmental disturbance, hardware failure and human impact etc. Figure 13 shows the performance of CP when none of this is taken into consideration. Positions can be constrained mistakenly into the wrong location.

ARCP is applied to provide the system with more adaptivity to varying situations. The positioning system has more freedom to adjust the "strength" of the required constraint as well as choose the optimal collaborative network. When a network with good geometry, sufficient signals and good accuracy measurement is selected, the relative constraint is "tighter" so that only particles lying within the threshold will remain and those outside will be killed, bringing the rover state estimation closer to the truth. A less ideal network will produce a "loose" constraint so that fewer particles would be killed to avoid pushing particles towards the wrong location.

Table 1 and Table 2 list the mean and maximum positioning error throughout Trial A and Trial B. Table 2 only list the error for Rover 1, as the ground truth for Rover 2 is provided by the UWB system which is not accurate enough to justify the positioning accuracy of ARCP. PDR indicates the result of DR from inertial measurements with wall constraint. CP indicates 
the result of non-adaptive $\mathrm{CP}$ with wall constraint. The $\mathrm{CP}$ result in Table 1 is an average of integrating one of the three anchors each time and the CP result in Table 2 is an average of integrating all available measurements.

In Trial B, as two rovers and four anchors are available, only the appropriate units are selected. As anchors are not represented by particles, therefore increasing the number of anchors does not affect the computation cost too much. However, the processing time is reduced by at least $5 \%$ when a rover is integrated. Hence the network size is kept within four, which was indicated as the effective size.

Table 1 Positioning errors for Trial A (NGB) (m)

\begin{tabular}{cccccccccc}
\hline & \multicolumn{2}{c}{ PDR } & \multicolumn{2}{c}{ CP } & \multicolumn{2}{c}{ ARCP (wall) } & \multicolumn{2}{c}{ ARCP (no wall) } \\
& mean & $\max$ & mean & $\max$ & mean & $\max$ & $\operatorname{mean}$ & $\max$ \\
\hline Rover 1 & 2.95 & 7.87 & 1.65 & 4.25 & 1.17 & 2.83 & 1.18 & 3.12 \\
Rover 2 & 1.27 & 3.76 & 1.05 & 4.40 & 0.71 & 1.71 & 0.70 & 2.24 \\
\hline
\end{tabular}

Table 2 Positioning errors for Trial B (BSS) (m)

\begin{tabular}{|c|c|c|c|c|c|c|}
\hline & \multicolumn{2}{|c|}{$\mathrm{CP}$} & \multicolumn{2}{|c|}{ ARCP (wall) } & \multicolumn{2}{|c|}{ ARCP (no wall) } \\
\hline & mean & $\max$ & mean & $\max$ & mean & $\max$ \\
\hline Rover 1 & 5.30 & 15.99 & 2.03 & 8.61 & 2.28 & 8.98 \\
\hline
\end{tabular}

As not enough factors are considered in CP, ARCP improves positioning accuracy by $25 \%$ in Trial A and 60\% in Trial B compared to CP. In Trial A, the improvement is more obvious for Rover 1 as the trajectory for Rover 2 is much simpler and the wall constraint is quite sufficient to constrain the inertial bias. The improvement is also much more obvious in Trial $\mathrm{B}$ where real ranging data is implemented, which are noisier and more unstable. ARCP can cope with different noise levels of real data with its threshold adjustment.

In both trials, the same threshold categories are applied as specified in Eq. (14). ARCP results demonstrate the ability to cope with situations without map information. Wall constraint is most effective in a straight long corridor without doors. However, such conditions are not always met and when the state predication model is noisy, wall constraints can misplace particles in the wrong room and restrict its chances of regenerating in the right location. Collaborative positioning can provide sufficient constraint even in places when wall constraint cannot. Therefore, the building map information can be eliminated in the ARCP algorithm. This means that users can start navigating in an environment where no prior information is available.

\section{CONCLUSIONS}

Collaborative positioning enhances positioning performance by forming a collaborative network that integrates available positioning information including $\mathrm{P} 2 \mathrm{P}$ ranging measurements between nearby rovers and anchors to constrain the measurement errors. Ranging measurements vary in different environments and conditions. If the wrong information is integrated, position estimation may be pushed further into the wrong location while reducing positioning efficiency unnecessarily. To avoid this, only the useful measurements are selected and integrated into the positioning system.

This paper proposes an adaptive ranging constraint collaborative positioning strategy that 
enables the user to decide on the most effective network at each epoch. This selection is based on the network geometry, network size and ranging accuracy of the units and their measurements. All three elements are combined to produce a decisive factor, MDOP, which helps the system to select the appropriate units as well as set the proper constraint threshold. Only those units that form a good geometry while providing high ranging accuracy will be used for positioning and others will be neglected. ARCP improves the positioning accuracy by more than $60 \%$ for real data, while reducing the maximum error by $45 \%$. The contribution of ranging constraints also enables the system to navigate when no interior building map is available.

By applying ARCP, the system produces results with higher accuracy and enhanced robustness. It allows the system to start up without prior information on the surrounding environment as long as collaborative units are found. This could be applied with Wi-Fi fingerprinting to introduce more adaptivity into the positioning system enabling it to cope with various difficult situations in the real world.

\section{REFERENCE}

Abdulrahim, K., Hide, C., Moore, T. and Hill, C. (2011). Aiding low cost inertial navigation with building heading for pedestrian navigation. The Journal of Navigation, 64, 219-233.

Arulampalam, M.S., Maskell, S., Gordon, N. and Clapp, T. (2002). A Tutorial on Particle Filters for Online Nonlinear/Non-Gaussian Bayesian Tracking. IEEE Transactions on Signal Processing, 50(2), 174-188.

Chen, C.S., Chiu, Y.J., Lee, C.T. and Lin, J.M. (2013). Calculation of Weighted Geometric Dilution of Precision. Journal of Applied Mathematics, 2013, 953048.

Cinefra, N. (2012) Adaptive Indoor Positioning System Based On Bluetooth Low Energy RSSI. Thesis, Politecnico Di Milano, Italy.

Evennou F. and Marx F. (2006). Advanced Integration of WiFi and Inertial Navigation Systems for Indoor Mobile Positioning. Eurasip Journal on Applied Signal Processing, 2006: 086706.

Gentile, C. and Kik, A. (2007). A Comprehensive Evaluation of Indoor Ranging using Ultra-Wideband Technology. EURASIP Journal on Wireless Communications and Networking, 2007, 86031.

Gustafsson, F., Gunnarsson, F., Bergman, N., Forssell, U., Jansson, J., Karlsson, R. and Nordlund, P.J. (2002). Particle Filters for Positioning, Navigation, and Tracking. IEEE Transactions on Signal Processing, 50(2), 425-437.

Ismail, G., Chong, C.C., Watanabe, F. and Inamura, H. (2008). NLOS identification and weighted least-squares localization for UWB systems using multipath channel statistics. EURASIP Journal on Advances in Signal Processing, 2008 (1), 271984.

Jing, H., Pinchin, J., Hill, C. and Moore, T. (2013). Wi-Fi Indoor Localisation Based on Collaborative Ranging Between Mobile Users. Proceedings of the $26^{\text {th }}$ International Technical Meeting of the ION Satellite Division, ION GNSS+2013, Nashville, Tennessee.

Kaemarungsi, K. and Krishnamurthy, P. (2012). Analysis of WLAN's Received Signal Strength Indication for Indoor Location Fingerprinting. Pervasive and Mobile Computing, 8, 292-316.

Koppanyi, Z., Toth, C.K., Grejner-Brzezinska, D.A. and Jozkow, G. (2014). Performance 

International Technical Meeting of The Institute of Navigation, ITM 2014, San Diego, California. UWB Radio Link. IEEE Journal on Selected Areas in Communications, 20(9), 16771683.

Marano, S., Gifford, W., Wymeersch, H. and Win, M.Z. (2010). NLOS identification and mitigation for localization based on UWB experimental data. IEEE Journal on Selected Areas in Communications, 28 (7): 1026-1035. Based Positioning for Indoor WLAN Systems. 2008 IEEE/ION Position, Location and Navigation Symposium, 685-688, Monterey, CA.

Patwari, N., Ash, J.N., Kyperountas, S., Hero, A.O., Moses, R.L. and Correal, N.S. (2005). Locating the Nodes: Cooperative Localization in Wireless Sensor Networks. IEEE Signal Processing Magazine, 22(4), 54-69.

Penna, F., Caceres, M.A. and Wymeersch, H. (2010). Cramér-Rao Bound for Hybrid GNSS-Terrestrial Cooperative Positioning, IEEE Communications Letters, 14(11), 10051007.

Pinchin, J., Hide, C. and Moore, T. (2012). A Particle Filter Approach to Indoor Navigation Using A Foot Mounted Inertial Navigation System and Heuristic Heading Information. 2012 International Conference on Indoor Positioning and Indoor Navigation (IPIN), Sydney, NSW.

Seo, S., Lee, B. (2010). Compact UWB Diversity Antenna for Mobile Phone Applications. 2010 Asia-Pacific Microwave Conference Proceedings (APMC), pp.22682270, Yokohama, Japan.

Tang, S., Kubo, N. and Ohashi, M. (2012). Cooperative Relative Positioning for Telecommunications, 506-511, Taipei, Taiwan.

von Watzdorf, S. and Michahelles, F. (2010). Accuracy of Positioning Data on Smartphones. Proceedings of the 3rd International Workshop on Location and the Web,

Xu, C., and Law, C.L. (2009). Experimental Evaluation of UWB Ranging Performance for Correlation and ED Receivers in Indoor Environments. International Journal of Hybrid Information Technology, 2(2), 37-54.

Weyn, M. and Schrooyen, F. (2008). A WiFi Assisted GPS Positioning Concept. ECUMICT, Gent, Belgium.

Wymeersch, H., Lien, J. and Win, M.Z. (2009). Cooperative Localization in Wireless Networks. Proceedings of the IEEE, 97(2), 427-450.

Wymeersch, H., Marano, S., Gifford, W.M. and Win, M.Z. (2012). A machine learning approach to ranging error mitigation for UWB Localization. IEEE Transactions on Communications, 60 (6): 1719-1728.

Yang, C. (2014). Covariance Analysis of Spatial and Temporal Effects of Collaborative Navigation. Proceedings of the 2014 IEEE/ION Position Location and Navigation 
514 Yang, C., Nguyen,T., Venable, D., White, L.M. and Siegel,R. (2009). Cooperative 515 Position Location with Signals of Opportunity. Proceedings of the IEEE 2009 National 516 Aerospace \& Electronics Conference (NAECON), Dayton, $\mathrm{OH}$.

517 Yao, J., Balaei, A.T., Hassan, M., Alam, N. and Dempster, A.G. (2011). Improving 518 Cooperative Positioning for Vehicular Networks. IEEE Transactions on Vehicular 519 Technology, 60(6), 2810-2823.

520 Zirari, S., Canalda, P. and Spies, F. (2009). Geometric and Signal Strength Dilution of 521 Precision (DoP)Wi-Fi. IJCSI International Journal of Computer Science Issues, 3, 35-44. 